\title{
Huge Hepatocellular Carcinoma Exhibiting a Complete Response after Stereotactic Body Radiation Therapy
}

\author{
Kyung In Shin', Byoung Kuk Jang' ${ }^{1}$ Jin Hee Kim², Jae Seok Hwang ${ }^{1}$ \\ Departments of ${ }^{1}$ Internal Medicine, ${ }^{2}$ Radiation Oncology, Keimyung University School of Medicine, Daegu, Korea
}

Received Dec. 30, 2019

Revised Feb. 25, 2020

Accepted Feb. 26, 2020
To date, there are limited data and little consensus on treatment strategies for huge hepatocellular carcinoma (HCC). Surgical resection provides significantly better survival than other modalities for single large HCC regardless of tumor stage. Recently, with technological advances in radiation therapy, stereotactic body radiation therapy (SBRT) is considered an alternative treatment option for HCC. Herein, we present a case of huge HCC that was successfully managed by SBRT. Transarterial embolization, previously performed in Russia, was incomplete. It was also not suitable for resection and transarterial chemoembolization. Although the rationale for radiotherapy in huge HCC was insufficient, SBRT was performed because no other treatment options were available. Additional radiofrequency ablation was performed for small HCC in a different segment, and radiological complete response (CR) was achieved. The CR was maintained over 4 years. Therefore, SBRT may be an alternative treatment option for large HCC that is not suitable for curative treatment. (J Liver Cancer 2020;20:167-172)

Keywords: Hepatocellular carcinoma; Stereotactic body radiotherapy

\section{INTRODUCTION}

Hepatocellular carcinoma (HCC) is the fifth commonest cancer and the second most frequent cause of cancer-related death globally. ${ }^{1}$ Treatment selection depends on tumor characteristics, the severity of underlying liver dysfunction, age, other medical comorbidities, financial state and available medical resources and local expertise. ${ }^{2}$ Currently, there are few data and little consensus on treatment strategies for huge HCC. A huge HCC is defined as a tumor with diameter $\geq 10 \mathrm{~cm}$

\section{Corresponding author: Jae Seok Hwang}

Department of Internal Medicine, Keimyung University School of Medicine, 1035 Dalgubeol-daero, Dalseo-gu, Daegu 42601, Korea

Tel. +82-53-258-4341, Fax. +82-53-258-4343

E-mail; gastro@dsmc.or.kr

https://orcid.org/0000-0002-1510-3534 and commonly surgical resection is regarded as the best treatment option for a single large HCC without vascular or bile duct invasion. However, in many cases, surgery is difficult due to deteriorated liver function or the location of the tumor. ${ }^{3}$ Nowadays, with remarkable technological advances in radiation therapy, stereotactic body radiation therapy (SBRT) has been regarded as an alternative treatment option for HCC that is not suitable for hepatic resection and radiofrequency ablation (RFA). ${ }^{4-6}$ Recently, the 2018 Korean Liver Cancer Association (KLCA)-National Cancer Center (NCC) Korea Practice Guidelines for HCC suggested that SBRT could be an alternative treatment to surgical resection or transarterial chemoembolization (TACE) in single large HCC. ${ }^{7}$ In this article, we report a case of huge HCC that was successfully managed with SBRT. As this article was based on protected health information, the need for Institutional Re- 
view Board and Ethics Committee approval was waived.

\section{CASE REPORT}

\section{Clinical findings}

A 57-year-old Russian man visited our outpatient clinic for the treatment of a huge hepatic mass detected on computed tomography (CT) scan. The patient had experienced intermittent right upper quadrant pain for 6 months. He was diagnosed with chronic hepatitis B virus infection 25 years prior, but did not follow-up regularly for surveillance of HCC. Initially, the patient visited a local hospital in Russia on account of right upper quadrant pain and was diagnosed with liver cirrhosis with splenomegaly and huge HCC. He underwent transarterial embolization for HCC at that hospital. However, the pain worsened gradually after treatment, so he visited our hospital for further treatment. Physical examination revealed right upper quadrant tenderness. On the initial complete blood count panel, the white blood cell count was $4.26 \times 10^{3} / \mu \mathrm{L}$, the hemoglobin level was $15.6 \mathrm{~g} / \mathrm{dL}$, and the platelet count was $218 \times 10^{3} / \mu \mathrm{L}$. A liver function test revealed a serum bilirubin level of $1.15 \mathrm{mg} / \mathrm{dL}$, an albumin level of $3.7 \mathrm{~g} / \mathrm{dL}$, a prothrombin time international normalized ratio of 1.36, an aspartate aminotransferase level of $105 \mathrm{IU} / \mathrm{L}$, and an alanine aminotransferase level of 53 IU/L. The hepatitis $\mathrm{B}$ virus was not detected on polymerase chain reaction assay, and the indocyanine green (ICG) R15 retention test yielded a level of $39.84 \%$. The serum level of alpha-fetoprotein (AFP) was $2,002.7 \mathrm{ng} / \mathrm{mL}$. A protein induced by vitamin $\mathrm{K}$ abscence or antagonist-II (PIVKA-II) test was not performed.

\section{Imaging findings}

An initial abdominal dynamic CT scan performed in Russia revealed a cirrhotic liver and a $12.2 \mathrm{~cm}$ wide hepatic mass in segment VIII-V, without any intrahepatic metastasis or lymph node enlargement. The hepatic mass was characteris-

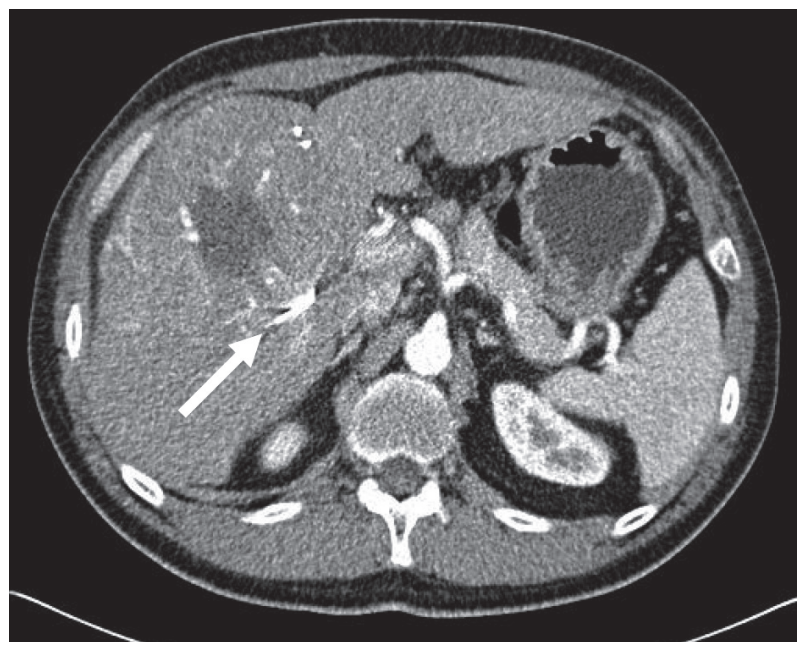

Figure 2. Abdominal computed tomography scan obtained at our hospital. The hepatic mass grew up to $13.3 \mathrm{~cm}$ and a long and radiopaque material was inserted in the hepatic artery (arrow); thereby, occlusion of hepatic artery was noted.
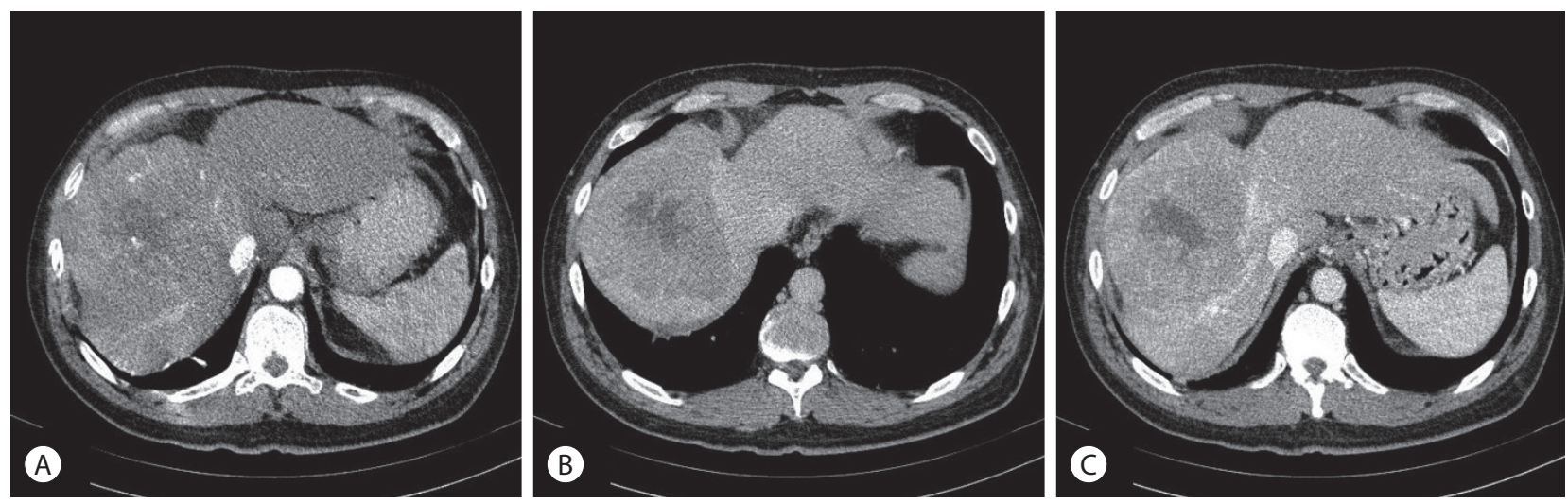

Figure 1. Initial dynamic computed tomography scan findings. A single $12.2 \mathrm{~cm}$ wide mass in segment VIII-V showed enhancement in the arterial phase (A), and washout in the portal-phase (B) and the delayed phase (C). 
tic of typical HCC with early arterial enhancement and portal venous washout (Fig. 1). One month after transarterial embolization treatment in Russia, the patient visited our hospital and underwent abdominal CT scan again. The hepatic mass enlarged up to a width of $13.3 \mathrm{~cm}$. A long and radiopaque material was inserted into the hepatic artery, whereby occlusion of the hepatic artery was noted (Fig. 2). Perhaps in Russia, the hepatic artery was blocked with a coil for the purposes of embolization. Both positron emission tomography CT scan and chest CT scan showed no extrahepatic tumor metastasis.

\section{Diagnosis and treatment}

Based on the clinical and imaging findings, the patient was diagnosed with a $13.3 \mathrm{~cm}$ wide HCC with neither portal vein invasion nor extrahepatic metastasis. He had a Child-Pugh score of 5 and a performance status score of 0 . Thus, the HCC was classified as an early stage tumor using the Barcelona Clinic Liver Cancer staging system, and as a stage II (T2, N0, M0) tumor according to the modified International
Union Against Cancer system. According to the 2018 KLCANCC Korea Practice Guidelines for HCC, the best option for this patient was resection. However, the patient had a financial problem and trouble following up postoperatively because he had to return to Russia after treatment. In addition, ICG test was 39.84\%, and there was concern about liver fail-

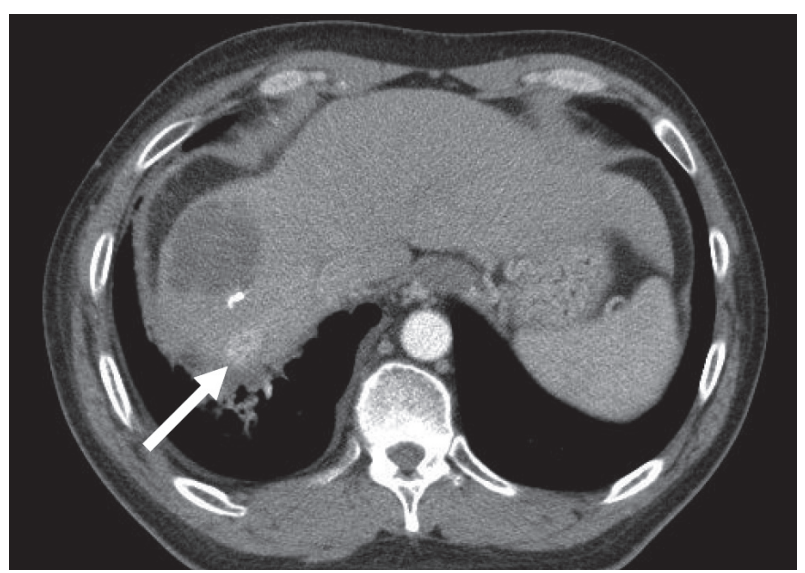

Figure 4. Findings on follow-up dynamic computed tomography scan 5 months after stereotactic body radiation therapy. There was no viable hepatocellular lar carcinoma in segment VIII-V, but intrahepatic metastasis was present in segment VII (arrow).

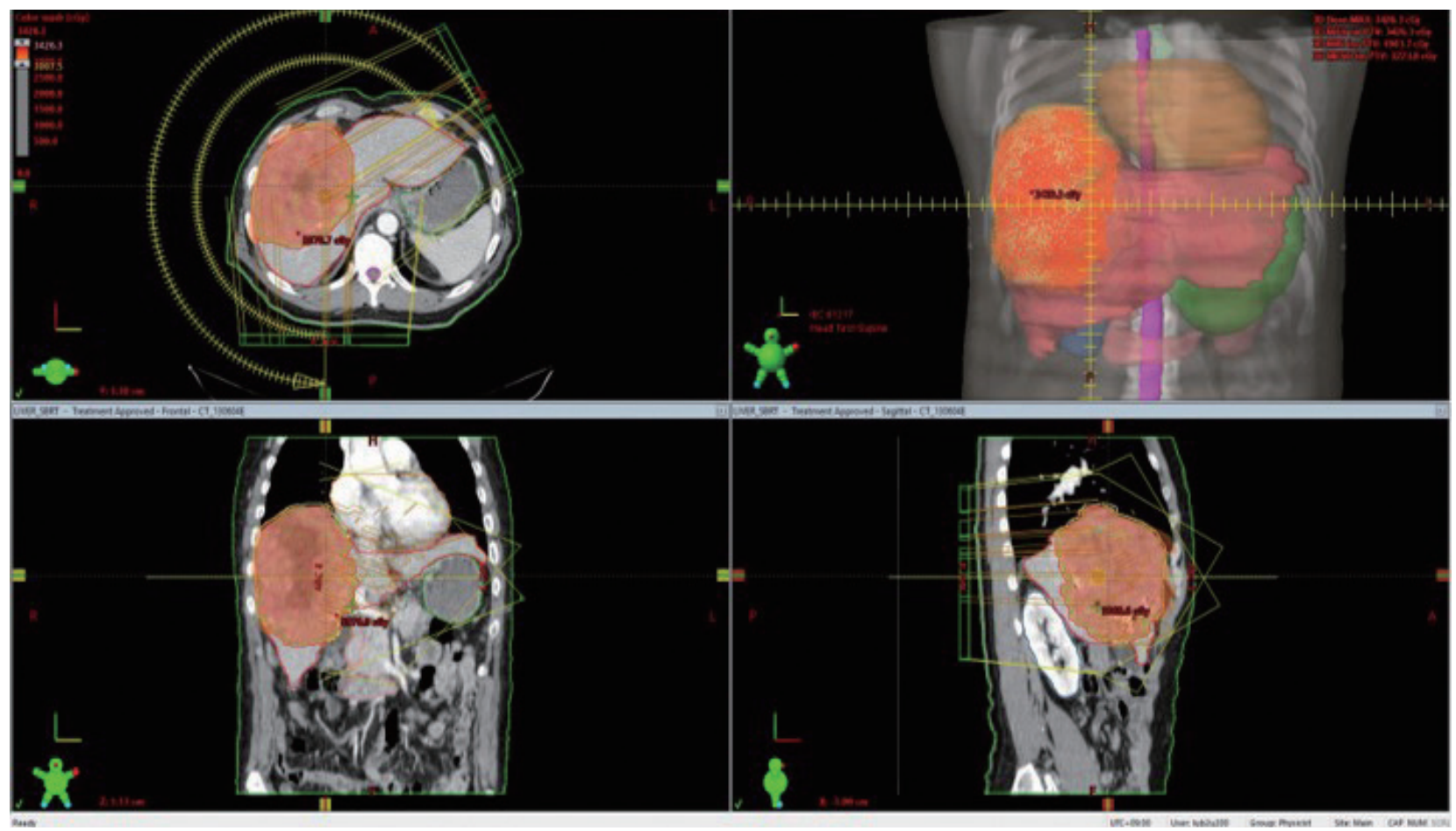

Figure 3. Planning computed tomography scan for stereotactic body radiation therapy. We planned to target the entire hepatocellular mass. 

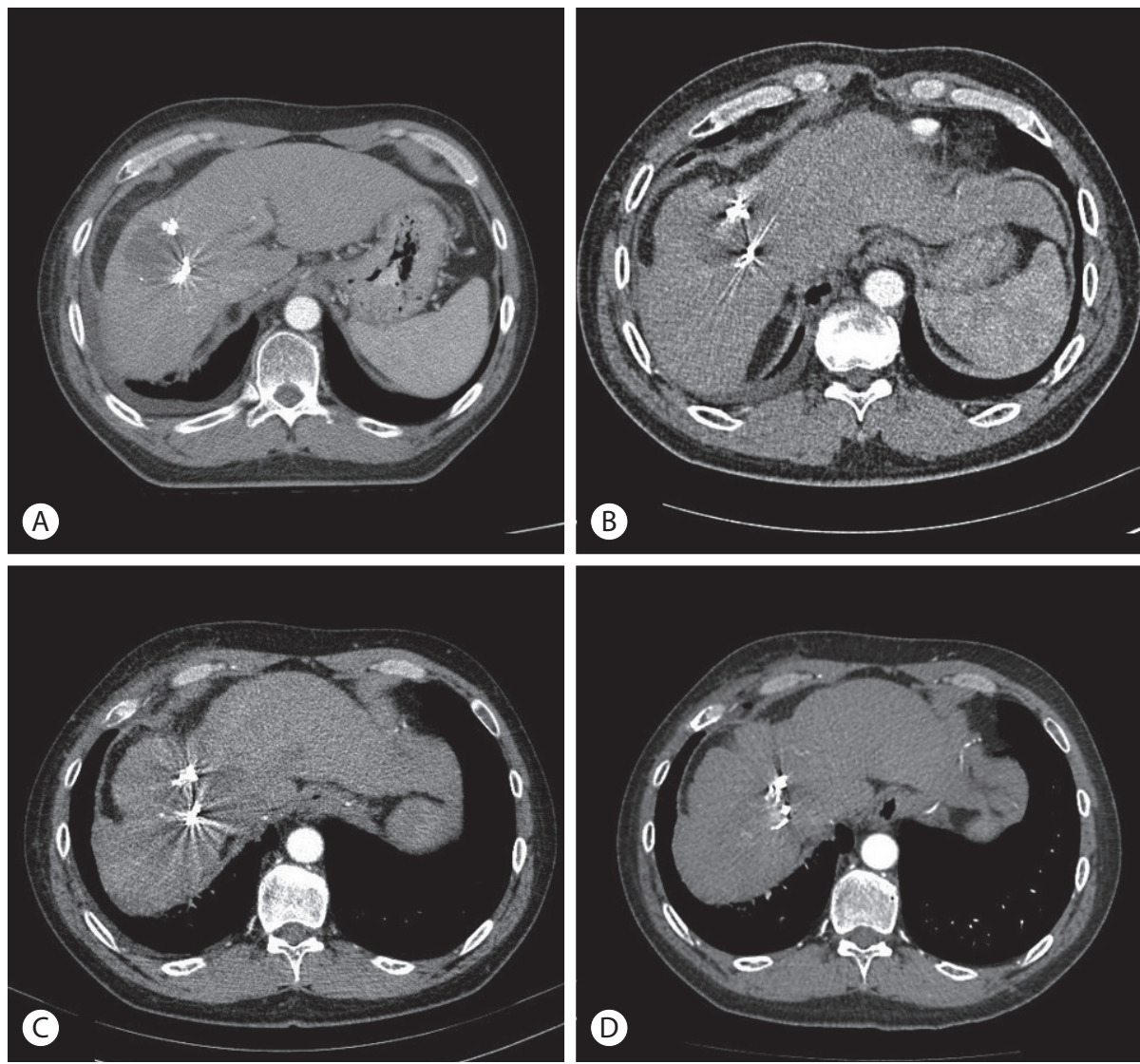

Figure 5. Findings on follow-up dynamic computed tomography scan after stereotactic body radiation therapy (SBRT). Necrotic change without any enhancement was observed at the tumor site 5 months after SBRT (A). The necrotic lesion gradually decreased in size, and the complete response status was maintained for 1 year (B), 2 years (C), and 4 years (D) after SBRT.

ure after surgery. The next option, TACE, could not be attempted because the hepatic artery was occluded. Therefore, we decided to perform SBRT in consultation with the Radiation Oncology Department. We planned to target the entire HCC mass and the SBRT was performed three times with 10 Gy (Fig. 3). After 3 months, a follow-up CT scan showed nearly complete necrosis of the HCC in segment VIII-V. Five months after SBRT, there is no viable HCC in segment VIIIV. However, in segment VII, a small mass with arterial enhancement and portal venous washout was detected; thus, RFA was performed on the small HCC (Fig. 4). After RFA, the tumor response was evaluated according to the modified Response Evaluation Criteria in Solid Tumors, ${ }^{8}$ and a complete response (CR) was identified. The serum levels of AFP decreased from 2,002.7 to $7.0 \mathrm{ng} / \mathrm{mL}$. Until now, he had maintained CR for over 4 years after SBRT (Fig. 5). There was concern about decreased liver function after radiotherapy, but liver function was well preserved as at the last followup.

\section{DISCUSSION}

Patients with huge HCC $\geq 10 \mathrm{~cm}$ in diameter represent a special subgroup for treatment. To date, there are limited data and little consensus on treatment strategies for huge HCC. ${ }^{9}$ Generally, patients with a single large HCC should be classified at least as having intermediate stage HCC. Surgical resection provides significantly better survival than does TACE for single large HCC regardless of tumor stage. ${ }^{10}$ TACE may be used in cases where such radical treatment is difficult. In some case, radiation therapy may also be a good alternative. ${ }^{11}$ Radiation therapy, however, has not been used 
as curative treatment for HCC. ${ }^{12}$ Most patients with HCC have underlying chronic liver disease and could not receive sufficient doses for the treatment of HCC. ${ }^{13}$ However, with advacements in technology and increasing clinical use, radiation therapy is considered reliable and effective for the treatment of HCC, depending on the extent of disease and characteristics of the patient. ${ }^{14}$ Radiation therapy can offer high local control rates in unresectable HCC, including cases with major vascular involvement, and can provide a modality to help bridge patients to potentially curative resection or transplantation. ${ }^{15}$ Recent studies report that SBRT has a local effect similar to surgery in small HCC with diamteter $<5 \mathrm{~cm}^{1{ }^{16}}$ However, as the size of the HCC increases, the local inhibition rate is reported to decrease. ${ }^{17}$

The limitation of SBRT was hepatotoxicity secondary to radiation. ${ }^{18}$ One study reported that SBRT is relatively safe in patients with hepatic function with a Child-Pugh score of 7 or less. ${ }^{19}$ In addition, Jun et al. ${ }^{20}$ reported that side effects after SBRT were very low in patients with a Child-Pugh score of 7 or less.

In this case, although the rationale for radiotherapy in huge HCC was insufficient, SBRT was performed because no other treatment options were available, and we achieved CR. There may have been an effect of transarterial embolization in Russia, but it would had been limited because the effect of embolization was insufficient. There was no change in tumor size after transarterial embolization but the tumor shrunk dramatically after SBRT. It seems to have a good response to stereotactic radiotherapy since the patient's performance was good and there was no major vascular invasion or extrahepatic metastasis. Surgical resection and TACE will still be a priority for huge HCC, but SBRT may be a good alternative treatment.

\section{AUTHOR CONTRIBUTIONS}

Drafting of the manuscript: Kyung In Shin (https://orcid. org/0000-0001-9311-8160). Critical revision of the manuscript for important intellectual content: Byoung Kuk Jang (https://orcid.org/0000-0002-8950-0866). Critical revision of the manuscript and final approval of the version to be pub- lished: Jae Seok Hwang (https://orcid.org/0000-0002-15103534).

\section{Conflicts of Interest}

The authors have no conflicts of interest to disclose.

\section{REFERENCES}

1. Bray F, Ferlay J, Soerjomataram I, Siegel RL, Torre LA, Jemal A. Global cancer statistics 2018: GLOBOCAN estimates of incidence and mortality worldwide for 36 cancers in 185 countries. CA Cancer J Clin 2018;68:394-424.

2. Yang JD, Hainaut $P$, Gores GJ, Amadou A, Plymoth A, Roberts LR. A global view of hepatocellular carcinoma: trends, risk, prevention and management. Nat Rev Gastroenterol Hepatol 2019;16:589604.

3. Kudo M, Izumi N, Kubo S, Kokudo N, Sakamoto M, Shiina S, et al. Report of the 20th nationwide follow-up survey of primary liver cancer in Japan. Hepatol Res 2020;50:15-46.

4. Ohri N, Dawson LA, Krishnan S, Seong J, Cheng JC, Sarin SK, et al. Radiotherapy for Hepatocellular Carcinoma: New Indications and Directions for Future Study. J Natl Cancer Inst 2016;108:djw133.

5. Rim CH, Seong J. Application of radiotherapy for hepatocellular carcinoma in current clinical practice guidelines. Radiat Oncol J 2016;34:160-167.

6. Citrin DE. Recent developments in radiotherapy. N Engl J Med 2017;377:2200-2201.

7. Korean Liver Cancer Association, National Cancer Center. 2018 Korean Liver Cancer Association-National Cancer Center Korea Practice Guidelines for the Management of Hepatocellular Carcinoma. Korean J Radiol 2019;20:1042-1113.

8. Lencioni R, Llovet JM. Modified RECIST (mRECIST) assessment for hepatocellular carcinoma. Semin Liver Dis 2010;30:52-60.

9. Xue T, Le F, Chen R, Xie X, Zhang L, Ge N, et al. Transarterial chemoembolization for huge hepatocellular carcinoma with diameter over ten centimeters: a large cohort study. Med Oncol 2015;32:64.

10. Liu PH, Su CW, Hsu CY, Hsia CY, Lee YH, Huang YH, et al. Solitary large hepatocellular carcinoma: staging and treatment strategy. PLoS One 2016;11:e0155588.

11. Sanuki N, Takeda A, Kunieda E. Role of stereotactic body radiation therapy for hepatocellular carcinoma. World J Gastroenterol 2014;20:3100-3111.

12. Cheng SH, Lin YM, Chuang VP, Yang PS, Cheng JC, Huang AT, et al. A pilot study of three-dimensional conformal radiotherapy in unresectable hepatocellular carcinoma. J Gastroenterol Hepatol 1999;14:1025-1033.

13. Lawrence TS, Robertson JM, Anscher MS, Jirtle RL, Ensminger WD, Fajardo LF. Hepatic toxicity resulting from cancer treatment. Int J 
Radiat Oncol Biol Phys 1995;31:1237-1248.

14. Choi SH, Seong J. Strategic application of radiotherapy for hepatocellular carcinoma. Clin Mol Hepatol 2018;24:114-134.

15. Chen CP. Role of radiotherapy in the treatment of hepatocellular carcinoma. J Clin Transl Hepatol 2019;7:183-190.

16. Su TS, Liang P, Liang J, Lu HZ, Jiang HY, Cheng T, et al. Long-term survival analysis of stereotactic ablative radiotherapy versus liver resection for small hepatocellular carcinoma. Int J Radiat Oncol Biol Phys 2017;98:639-646.

17. Scorsetti M, Comito T, Cozzi L, Clerici E, Tozzi A, Franzese C, et al. The challenge of inoperable hepatocellular carcinoma (HCC): results of a single-institutional experience on stereotactic body radiation therapy (SBRT). J Cancer Res Clin Oncol 2015;141:1301-
1309.

18. Pan CC, Kavanagh BD, Dawson LA, Li XA, Das SK, Miften M, et al. Radiation-associated liver injury. Int J Radiat Oncol Biol Phys 2010;76(3 Suppl):S94-S100.

19. Huertas A, Baumann AS, Saunier-Kubs F, Salleron J, Oldrini G, Croisé-Laurent $V$, et al. Stereotactic body radiation therapy as an ablative treatment for inoperable hepatocellular carcinoma. Radiother Oncol 2015;115:211-216.

20. Jun BG, Kim YD, Cheon GJ, Kim ES, Jwa E, Kim SG, et al. Clinical significance of radiation-induced liver disease after stereotactic body radiation therapy for hepatocellular carcinoma. Korean J Intern Med 2018;33:1093-1102. 